\title{
Extreme Tenacity of Graphs with Given Order and Size
}

\author{
T. C. E. Cheng $\cdot$ Yin-Kui Li $\cdot$ Chuan-Dong Xu • \\ Sheng-Gui Zhang
}

Received: 6 April 2014/Revised: 25 July 2014/Accepted: 28 July 2014/

Published online: 14 August 2014

(C) Operations Research Society of China, Periodicals Agency of Shanghai University, and SpringerVerlag Berlin Heidelberg 2014

\begin{abstract}
Computer or communication networks are so designed that they do not easily get disrupted under external attack and, moreover, these are easily reconstructible if they do get disrupted. These desirable properties of networks can be measured by various graph parameters, such as connectivity, toughness, scattering number, integrity, tenacity, rupture degree and edge-analogues of some of them. Among these parameters, the tenacity and rupture degree are two better ones to measure the stability of a network. In this paper, we consider two extremal problems on the tenacity of graphs: determine the minimum and maximum tenacity of graphs with given order and size. We give a complete solution to the first problem, while for the second one, it turns out that the problem is much more complicated than that of the minimum case. We determine the maximum tenacity of trees with given order
\end{abstract}

\footnotetext{
This work was supported by National Natural Science Foundation of China (No. 11271300), the Open Fund of Xi'an Jiaotong University, China (No. 2010-4) and the Fund of Qinghai University for Nationalities (xjz201403).
}

\section{T. C. E. Cheng}

Department of Logistics and Maritime Studies, The Hong Kong Polytechnic University, Hung Hom, Kowloon, Hong Kong, China

\section{Y.-K. Li}

School of Mathematics and Statistics, Qinghai University for Nationalities, Xining 810000, Qinghai, China

C.-D. Xu - S.-G. Zhang

Department of Applied Mathematics, Northwestern Polytechnical University,

Xi'an 710072, Shaanxi, China

S.-G. Zhang $(\bowtie)$

State Key Laboratory for Manufacturing Systems Engineering, Xi'an Jiaotong University, Xi' an 710049, Shaanxi, China e-mail: sgzhang@nwpu.edu.cn 
and show the corresponding extremal graphs. The paper concludes with a discussion of a related problem on the edge vulnerability parameters of graphs.

Keywords Vulnerability parameters - Minimum tenacity - Maximum tenacity · Trees

\section{Introduction}

In the analysis of the vulnerability of networks to disruption, three quantities (there may be others) came to mind, i.e., (1) the number of elements that are not functioning; (2) the number of remaining connected subnetworks and (3) the size of a largest remaining group within which mutual communication can still occur. Based on these quantities, many graph parameters, such as connectivity, toughness [5], scattering number [14], integrity [1], tenacity [6], rupture degree [15] and edge-analogues of some of them, have been proposed for measuring the vulnerability of networks.

Throughout the paper, we use Bondy and Murty [3] for terminology and notation not defined here. For a graph $G$, by $\omega(G)$ we denote the number of components of $G$, and $\tau(G)$ the order of a largest component of $G$. We shall use $\lfloor x\rfloor$ for the largest integer not larger than $x$ and $\lceil x\rceil$ the smallest integer not smaller than $x$.

The connectivity is a parameter defined based on Quantity (1). The connectivity of a noncomplete graph $G$ is defined by

$$
\kappa(G)=\min \{|X|: X \subset V(G), \omega(G-X)>1\},
$$

and that of the complete graph $K_{n}$ is defined as $n-1$.

Both toughness and scattering number take into account Quantities (1) and (2). The toughness and scattering number of a noncomplete connected graph $G$ are defined by

$$
t(G)=\min \left\{\frac{|X|}{\omega(G-X)}: X \subset V(G), \omega(G-X)>1\right\}
$$

and

$$
s(G)=\max \{\omega(G-X)-|X|: X \subset V(G), \omega(G-X)>1\},
$$

respectively.

The integrity is defined based on Quantities (1) and (3). The integrity of a graph $G$ is defined by

$$
I(G)=\min \{|X|+\tau(G-X): X \subset V(G)\} .
$$

Both the tenacity and rupture degree take into account all the three quantities. The tenacity and rupture degree of a noncomplete connected graph $G$ are defined by

$$
T(G)=\min \left\{\frac{|X|+\tau(G-X)}{\omega(G-X)}: X \subset V(G), \omega(G-X)>1\right\}
$$

and 


$$
r(G)=\max \{\omega(G-X)-|X|-\tau(G-X): X \subset V(G), \omega(G-X)>1\},
$$

respectively.

From the above definitions, we can see that the connectivity of a graph reflects the difficulty in breaking down a network into several pieces. This invariant is often too weak, since it does not take into account what remains after the corresponding graph is disconnected. Unlike the connectivity, each of the other vulnerability measures, i.e., toughness, scattering number, integrity, tenacity and rupture degree, reflects not only the difficulty in breaking down the network but also the damage that has been caused. Further, we can easily see that the tenacity and rupture degree are the two most advanced ones among these parameters when measuring the stability of networks.

When designing stable networks, it is often required to know the structure of networks attaining the maximum and minimum values of given stability parameters with prescribed number of communications stations and links. This problem was first studied by the well-known graph theorist Harary [13]. Harary stated that among all the graphs with $n$ vertices and $m$ edges, the maximum connectivity is 0 when $m<n-1$ and $\left\lfloor\frac{2 m}{n}\right\rfloor$ when $m \geqslant n-1$. For two integers $n$ and $m$ with $n \leqslant m \leqslant\left(\begin{array}{l}n \\ 2\end{array}\right)$, Harary constructed graphs with $n$ vertices, $m$ edges and connectivity $\left\lfloor\frac{2 m}{n}\right\rfloor$, which are now widely known as the Harary graphs. Harary [13] also considered the minimum connectivity of graphs with a given number of vertices and edges. He showed that among all the graphs with $n$ vertices and $m$ edges, the minimum connectivity is 0 or $m-\left(\begin{array}{c}n-1 \\ 2\end{array}\right)$, whichever is larger. This lower bound on the connectivity can be achieved by any graph consisting of a complete subgraph $K_{n-1}$, together with exactly one additional vertex that is adjacent to any $m-\left(\begin{array}{c}n-1 \\ 2\end{array}\right)$ vertices of $K_{n-1}$.

As to connectivity, it is natural to ask what are the extreme values for each of these new vulnerability parameters of a graph with a given number of vertices and edges. We list the results on the extreme values of these vulnerability parameters of graphs in Table 1.

In this paper, we consider the problem of determining the extreme tenacity of a graph with a given number of vertices and edges. We give a complete solution to the problem for the minimum case in Sect. 2. The problem for the maximum case is much more complicated. In Sect. 3, we give a partial solution to this problem by determining the maximum tenacity of trees with given number of vertices and show

Table 1 Achievements of the study for extreme values of vulnerability parameters

\begin{tabular}{lll}
\hline & Maximum value & Minimum value \\
\hline (Edge-)Connectivity & Complete solution [13] & Complete solution [13] \\
Toughness & Partial solution [7-11] & Unknown \\
Scattering number & Complete solution [17] & Complete solution [17] \\
Integrity & Partial solution [2] & Complete solution [16] \\
Tenacity & Unknown & Unknown \\
Rupture degree & Unknown & Unknown \\
\hline
\end{tabular}


the corresponding extremal graphs. We conclude the paper with a discussion of a related problem on the edge-vulnerability parameters of graphs in the final section.

\section{Minimum Tenacity of Graphs}

Theorem 2.1 Let $n$ and $m$ be two positive integers with $n-1 \leqslant m \leqslant$ $\left(\begin{array}{l}n \\ 2\end{array}\right)-1$. Then among all the connected graphs with $n$ vertices and $m$ edges, the minimum tenacity is $\frac{k+1}{n-k}$, where $\left(\begin{array}{l}k \\ 2\end{array}\right)+(n-k)(k-1)<m \leqslant\left(\begin{array}{l}k \\ 2\end{array}\right)+(n-k) k$.

Proof Suppose that $G$ is a connected graph with $n$ vertices and $m$ edges such that its tenacity is minimum. Let $X^{*}$ be a vertex cut of $G$ with

$$
T(G)=\frac{\left|X^{*}\right|+\tau\left(G-X^{*}\right)}{\omega\left(G-X^{*}\right)} .
$$

We assume that $X^{*}$ is chosen such that $\left|X^{*}\right|$ is as large as possible. Denote the components of $G-X^{*}$ with at least two vertices by $G_{1}, G_{2}, \cdots, G_{p}$ and the components with only one vertices by $w_{1}, w_{2}, \cdots, w_{q}$.

Suppose $p \geqslant 2$. Choose a vertex $u_{i}$ in $G_{i}$ such that $u_{i}$ is adjacent to at least one vertex of $X^{*}$ for each $i$ with $1 \leqslant i \leqslant p$. Replace each edge set $\left\{u_{i} v: u_{i} v \in E\left(G_{i}\right), v \in\right.$ $\left.V\left(G_{i}\right)\right\}$ by a new edge set $\left\{u_{p} v: u_{i} v \in E\left(G_{i}\right), v \in V\left(G_{i}\right)\right\}$ for every $i$ with $1 \leqslant i \leqslant p-1$. Denote the resulting graph by $G^{\prime}$. Then $G^{\prime}$ is also a connected graph with $n$ vertices and $m$ edges, and $X^{\prime}=X^{*} \cup\left\{u_{p}\right\}$ is a vertex cut of $G^{\prime}$. The components of $G^{\prime}-X^{\prime}$ are consisted of the components of $G_{1}-u_{1}, G_{2}-u_{2}, \cdots, G_{p}-$ $u_{p}$ together with $w_{1}, w_{2}, \cdots, w_{q}$ and $u_{1}, u_{2}, \cdots, u_{p-1}$. Thus

$$
\omega\left(G^{\prime}-X^{\prime}\right) \geqslant \omega\left(G-X^{*}\right)+(p-1) \geqslant \omega\left(G-X^{*}\right)+1
$$

and

$$
\tau\left(G^{\prime}-X^{\prime}\right) \leqslant \tau\left(G-X^{*}\right)-1
$$

This implies that

$$
\begin{aligned}
T\left(G^{\prime}\right) & \leqslant \frac{\left|X^{\prime}\right|+\tau\left(G^{\prime}-X^{\prime}\right)}{\omega\left(G^{\prime}-X^{\prime}\right)} \\
& \leqslant \frac{\left|X^{*}\right|+1+\tau\left(G-X^{*}\right)-1}{\omega\left(G-X^{*}\right)+1} \\
& <\frac{\left|X^{*}\right|+\tau\left(G-X^{*}\right)}{\omega\left(G-X^{*}\right)} \\
& =T(G),
\end{aligned}
$$

a contradiction.

Suppose now $p=1$. We distinguish two cases.

Case $1 V\left(G_{1}\right)$ is a clique.

Let $u_{1}$ be a vertex in $G_{1}$. Set $X^{* *}=X^{*} \cup\left(V\left(G_{1}\right) \backslash\left\{u_{1}\right\}\right)$. Clearly we have

$$
\tau\left(G-X^{* *}\right)=1 \text { and } \omega\left(G-X^{* *}\right)=\omega\left(G-X^{*}\right) .
$$

Then $X^{* *}$ is a vertex cut of $G$ with 


$$
\left|X^{* *}\right|=\left|X^{*}\right|+\tau\left(G-X^{*}\right)-1
$$

and

$$
\frac{\left|X^{* *}\right|+\tau\left(G-X^{* *}\right)}{\omega\left(G-X^{* *}\right)}=\frac{\left|X^{*}\right|+\tau\left(G-X^{*}\right)}{\omega\left(G-X^{*}\right)}=T(G),
$$

contradicting the choice of $X^{*}$.

Case $2 V\left(G_{1}\right)$ is not a clique.

In this case, let $X_{1}$ be a vertex cut of $G_{1}$. Set $X^{* *}=X^{*} \cup X_{1}$. Then $X^{* *}$ is a vertex cut of $G$ with

$$
\left|X^{* *}\right|=\left|X^{*}\right|+\left|X_{1}\right|, \tau\left(G-X^{* *}\right) \leqslant \tau\left(G-X^{*}\right)-\left|X_{1}\right|-1,
$$

and

$$
\omega\left(G-X^{* *}\right) \geqslant \omega\left(G-X^{*}\right)+1
$$

This implies that

$$
\begin{aligned}
\frac{\left|X^{* *}\right|+\tau\left(G-X^{* *}\right)}{\omega\left(G-X^{* *}\right)} & \leqslant \frac{\left|X^{*}\right|+\left|X_{1}\right|+\tau\left(G-X^{*}\right)-\left|X_{1}\right|-1}{\omega\left(G-X^{*}\right)+1} \\
& <\frac{\left|X^{*}\right|+\tau\left(G-X^{*}\right)}{\omega\left(G-X^{*}\right)} \\
& =T(G),
\end{aligned}
$$

contradicting the definition of the tenacity of $G$.

From the above discussion, we have $p=0$, i.e., $\tau\left(G-X^{*}\right)=1$.

Now let $\left|X^{*}\right|=x$. Then

$$
T(G)=\frac{\left|X^{*}\right|+\tau\left(G-X^{*}\right)}{\omega\left(G-X^{*}\right)}=\frac{x+1}{n-x} .
$$

We claim that $x \geqslant k$. Otherwise,

$$
\begin{aligned}
m & =|E(G)| \leqslant\left(\begin{array}{l}
x \\
2
\end{array}\right)+(n-x) x \\
& =-\frac{1}{2}\left[x-\left(n-\frac{1}{2}\right)\right]^{2}+\frac{1}{2}\left(n-\frac{1}{2}\right)^{2} \\
& \leqslant-\frac{1}{2}\left[k-1-\left(n-\frac{1}{2}\right)\right]^{2}+\frac{1}{2}\left(n-\frac{1}{2}\right)^{2} \\
& =\left(\begin{array}{c}
k-1 \\
2
\end{array}\right)+(n-k+1)(k-1) \\
& =\left(\begin{array}{c}
k \\
2
\end{array}\right)+(n-k)(k-1),
\end{aligned}
$$

a contradiction.

Therefore, we have 


$$
T(G)=\frac{x+1}{n-x} \geqslant \frac{k+1}{n-k} .
$$

On the other hand, since $\left(\begin{array}{l}k \\ 2\end{array}\right)+(n-k)(k-1)<m \leqslant\left(\begin{array}{l}k \\ 2\end{array}\right)+(n-k) k$, it is easy to construct a connected graph with $n$ vertices and $m$ edges such that its tenacity is

$$
\frac{k+1}{n-k}
$$

which completes the proof of the theorem.

From the proof of Theorem 2.1, we deduce the following result:

Corollary 2.2 Let $n$ and $m$ be two positive integers with $n-1 \leqslant m \leqslant\left(\begin{array}{l}n \\ 2\end{array}\right)-1$, and $G$ be a connected graph with $n$ vertices and $m$ edges such that its tenacity is minimum. Then $G$ is such a graph that it contains an empty subgraph with order $n-k$, where $\left(\begin{array}{l}k \\ 2\end{array}\right)+(n-k)(k-1)<m \leqslant\left(\begin{array}{l}k \\ 2\end{array}\right)+(n-k) k$.

\section{Maximum Tenacity of Graphs}

In this section we consider the maximum tenacity of connected graphs with given number of vertices and edges. It turns out that this problem is much more complicated than that of the minimum case. Here we give the results for the problem involving trees.

In the following by an odd (or even) path we mean a path with an odd (or even) number of vertices. We first list some lemmas.

Lemma 3.1 (Cozzen et al. [6]) Let $G$ be a noncomplete connected graph and $H$ be a connected spanning subgraph of $G$. Then $T(H) \leqslant T(G)$.

Lemma 3.2 Let $G$ be a connected graph. If there exists a vertex cut $X_{0}$ of $G$ such that $G-X_{0}$ is a forest and $\omega\left(G-X_{0}\right) \geqslant\left|X_{0}\right|+2\left(\right.$ resp. $\left.\omega\left(G-X_{0}\right) \geqslant\left|X_{0}\right|+3\right)$, then $T(G) \leqslant 1$ (resp. $T(G)<1)$.

Proof If the maximum degree of $G-X_{0}$ is at most 1, then let $X^{*}=X_{0}$. Otherwise, choose a vertex $v_{1} \in V\left(G-X_{0}\right)$ with $d_{G-X_{0}}\left(v_{1}\right) \geqslant 2$ and set $X_{1}=X_{0} \cup\left\{v_{1}\right\}$. Then we have $\omega\left(G-X_{1}\right) \geqslant\left|X_{1}\right|+2$. Repeating this process, we can finally obtain a vertex cut $X_{k}$ of $G$ with $\omega\left(G-X_{k}\right) \geqslant\left|X_{k}\right|+2$ and the maximum degree of $G-X_{k}$ is at most 1 . Choose $X_{k}$ as $X^{*}$. Then we have

$$
\frac{\left|X^{*}\right|+\tau\left(G-X^{*}\right)}{\omega\left(G-X^{*}\right)} \leqslant \frac{\omega\left(G-X^{*}\right)-2+2}{\omega\left(G-X^{*}\right)}=1,
$$

which implies that $T(G) \leqslant 1$ by the definition of tenacity. The other assertion can be proved similarly.

Lemma 3.3 (Choudum and Priya [4]) The tenacity of the path $P_{n}$ is 


$$
T\left(P_{n}\right)=\left\{\begin{array}{cl}
1, & \text { if } n \text { is odd } \\
\frac{n+2}{n}, & \text { if } n \text { is even } .
\end{array}\right.
$$

Theorem 3.4 Among all the trees on $n$ vertices, let $G$ have the maximum tenacity. Then

(i) $G$ is a path when $n$ is even;

(ii) the maximum degree of $G$ is at most 3 and it contains no nonadjacent vertices of degree 3 when $n$ is odd.

Proof (i) Suppose that $G$ has the maximum tenacity among all trees on $n$ vertices and contains a vertex of degree at least 3, say $v_{0}$. Let $X_{0}=\left\{v_{0}\right\}$. Then $X_{0}$ is a vertex cut of $G$ such that $G-X_{0}$ is a forest and $\omega\left(G-X_{0}\right) \geqslant\left|X_{0}\right|+2$. It follows from Lemmas 3.2 and 3.3 that $T(G) \leqslant 1<T\left(P_{n}\right)$, a contradiction. This implies that $\Delta(G) \leqslant 2$ and $G$ is a path.

(ii) Suppose that $G$ has $n$ (is odd) vertices and contains a vertex of degree at least 4 , say $v_{0}$; or two nonadjacent vertices of degree 3 , say $u_{0}$ and $v_{0}$. Let $X_{0}=\left\{v_{0}\right\}$ or $X_{0}=\left\{u_{0}, v_{0}\right\}$. Then $X_{0}$ is a vertex cut such that $G-X_{0}$ is a forest and $\omega\left(G-X_{0}\right) \geqslant\left|X_{0}\right|+3$. It follows from Lemma 3.2 that $T(G)<1$.

Now let $G$ be a tree on $n$ (is odd) vertices with maximum degree 3 and contains no nonadjacent vertices of degree 3 . Note that since $G$ has no nonadjacent vertices of degree $3, G$ contains at most two vertices of degree 3 . We will show that $T(G)=1$.

Case 1 The maximum degree of $G$ is at most 2 .

Then $G$ is a path, and it follows from Lemma 3.3 that $T(G)=1$.

Case $2 G$ has only one vertex of degree 3 .

Let $u$ be the vertex of degree 3 and $X$ be an arbitrary vertex cut of $G$. If $u \notin X$, or $u \in X$ and $X$ contains two adjacent vertices of $G-u$ or a leaf of $G-u$, then we have $\omega(G-X) \leqslant|X|+1$, and therefore,

$$
\frac{|X|+\tau(G-X)}{\omega(G-X)} \geqslant \frac{|X|+\tau(G-X)}{|X|+1} \geqslant 1 .
$$

If $u \in X$ and $X$ contains neither adjacent vertices nor leaves of $G-u$, then we can see that $\omega(G-X)=|X|+2$ and at least one of the three components of $G-u$ is an even path (because $G$ has $n$ vertices and $n$ is odd), say $P_{\mathrm{e}}$. It is not difficult to see that

$$
\tau(G-X) \geqslant \tau\left(P_{\mathrm{e}}-X \cap V\left(P_{\mathrm{e}}\right)\right) \geqslant 2 .
$$

Therefore,

$$
\frac{|X|+\tau(G-X)}{\omega(G-X)} \geqslant \frac{\omega(G-X)-2+\tau\left(P_{\mathrm{e}}-X \cap V\left(P_{\mathrm{e}}\right)\right)}{\omega(G-X)} \geqslant 1 .
$$

On the other hand, since $G$ is a bipartite graph, the bipartition of $V(G)$ can be denoted by $[X, Y]$, where $u \in X$. Let 


$$
X^{*}=\{v \in X: d(v) \geqslant 2\} .
$$

Note that all those vertices in $X^{*}-u$ have degree 2 in $G$. It follows that $\tau(G-$ $\left.X^{*}\right) \leqslant 2$ and $\omega\left(G-X^{*}\right)=\left|X^{*}\right|+2$. Thus

$$
\frac{\left|X^{*}\right|+\tau\left(G-X^{*}\right)}{\omega\left(G-X^{*}\right)} \leqslant \frac{\left|X^{*}\right|+2}{\left|X^{*}\right|+2}=1 .
$$

From the above discussion and the definition of tenacity we can see that $T(G)=1$.

Case $3 G$ has exactly two adjacent vertices of degree 3 .

Let $u$ and $v$ be the two adjacent vertices of degree 3 and $X$ be an arbitrary vertex cut of $G$. First assume $|X \cap\{u, v\}| \leqslant 1$, say $u \in X$. Note that all the three components of $G-u$ are paths. By using the same method in Case 2, it can be proved that

$$
\frac{|X|+\tau(G-X)}{\omega(G-X)} \geqslant 1 .
$$

So now we assume that $|X \cap\{u, v\}|=2$. If $X$ contains adjacent vertices of $G-$ $\{u, v\}$ or a leaf of $G-\{u, v\}$, then $\omega(G-X) \leqslant|X|+1$, and therefore,

$$
\frac{|X|+\tau(G-X)}{\omega(G-X)} \geqslant \frac{|X|+\tau(G-X)}{|X|+1} \geqslant 1 .
$$

Otherwise, we have $\omega(G-X)=|X|+2$. Moreover, at least one of the three components of $G-u$ is an even path, say $P_{\mathrm{e}}$. If $v \notin V\left(P_{\mathrm{e}}\right), P_{\mathrm{e}}$ is an even path in $G-\{u, v\}$. Let $P_{\mathrm{e}}^{*}=P_{\mathrm{e}}$. If $v \in V\left(P_{\mathrm{e}}\right)$, then the two components of $P_{\mathrm{e}}-v$ have an odd number of vertices. One of them must be an even path, which is also an even path in $G-\{u, v\}$, denote it by $P_{\mathrm{e}}^{*}$. As above, we have

$$
\tau(G-X) \geqslant \tau\left(P_{\mathrm{e}}^{*}-X \cap V\left(P_{\mathrm{e}}^{*}\right)\right) \geqslant 2
$$

Therefore,

$$
\frac{|X|+\tau(G-X)}{\omega(G-X)} \geqslant \frac{\omega(G-X)-2+2}{\omega(G-X)}=1 .
$$

On the other hand, since $G$ is a bipartite graph, the bipartition of $V(G)$ can be denoted by $[X, Y]$, where $u \in X$. Let

$$
X^{*}=\{v \in X: d(v) \geqslant 2\} .
$$

Note that all those vertices in $X^{*}-u$ have degree 2 in $G$. It follows that $\tau(G-$ $\left.X^{*}\right) \leqslant 2$ and $\omega\left(G-X^{*}\right)=\left|X^{*}\right|+2$. Thus

$$
\frac{\left|X^{*}\right|+\tau\left(G-X^{*}\right)}{\omega\left(G-X^{*}\right)} \leqslant \frac{\left|X^{*}\right|+2}{\left|X^{*}\right|+2}=1 .
$$

From the above discussion and the definition of tenacity we can see that $T(G)=1$.

The proof is complete.

Corollary 3.5 Among all the trees on $n$ vertices, the maximum tenacity is 


$$
\left\{\begin{array}{cl}
1, & \text { if } n \text { is odd } \\
\frac{n+2}{n}, & \text { if } n \text { is even } .
\end{array}\right.
$$

\section{Extreme Values of Edge Vulnerability Parameters}

As we noted in Sect. 1, besides the vertex vulnerability parameters, the edgeanalogues of some of them have also been proposed, e.g., edge-toughness [12] for toughness, edge-integrity [1] for integrity, edge-tenacity [18] for tenacity. As for the vertex vulnerability parameters, it would be an interesting problem to determine the extreme values of the edge vulnerability parameters of graphs with a given number of vertices and edges.

Acknowledgments The authors are grateful to the anonymous referees for their helpful comments on the earlier version of this article.

\section{References}

[1] Barefoot, C.A., Entringer, R., Swart, H.: Vulnerability in graphs - a comparative survey. J. Combin. Math. Combin. Comput. 1, 12-22 (1987)

[2] Barefoot, C.A., Entringer, R., Swart, H.: Integrity of trees and powers of cycles. Congr. Numer. 58, 103-114 (1987)

[3] Bondy, J.A., Murty, U.S.R.: Graph Theory with Applications. Macmillan/Elsevier, London/New York (1976)

[4] Choudum, S.A., Priya, N.: Tenacity of complete graph products and grids. Networks 34, 192-196 (1999)

[5] Chvátal, V.: Tough graphs and Hamiltonian circuits. Discr. Math. 5, 215-228 (1973)

[6] Cozzen, M., Moazzami, D., Stueckle, S.: The tenacity of a graph. In: Proceedings of Seventh International Conference on the Theory and Applications of Graphs. Wiley, New York, pp. 1111-1122 (1995)

[7] Doty, L.L.: A large class of maximally tough graphs. OR Spektrum 13, 147-151 (1991)

[8] Doty, L.L., Ferland, K.K.: Supertough graphs need not be $K_{1,3}$-free. Australas. J. Combin. 32, 91-103 (2005)

[9] Doty, L.L., Ferland, K.K.: Some maximally tough circulants. Ars Combin. 87, 193-203 (2008)

[10] Ferland, K.K.: Maximum toughness among $(n, m)$-graphs. J. Combin. Math. Combin. Comput. 43, 43-55 (2002)

[11] Goddard, W.D., Swart, H.C.: On some extremal problems in connectivity. Graph Theory Combin. Appl. 1, 535-551 (1991)

[12] Gusfield, D.: Connectivity and edge disjoint spanning trees. Inf. Process. Lett. 16, 87-89 (1983)

[13] Harary, F.: The maximum connectivity of a graph. Proc. Natl Acad. Sci. U.S.A. 48, 1142-1146 (1962)

[14] Jung, H.A.: On a class of posets and the corresponding comparability graphs. J. Combin. Theory Ser. B 24, 125-133 (1978)

[15] Li, Y., Zhang, S., Li, X.: Rupture degree of graphs. Int. J. Comput. Math. 82, 793-803 (2005)

[16] Ma, R., Liu, N.: Minimum integrity of graphs. Acta Math. Sci. Ser. A 26, 251-257 (2006)

[17] Ouyang, K.: The relative breaktivity of graphs. Master's Degree Thesis, Lanzhou University, China (1987)

[18] Piazza, B.L., Robertst, F.S., Stueckle, S.K.: Edge-tenacious networks. Networks 25, 7-17 (1995) 